OPEN ACCESS

Edited by:

Noriyoshi Usui,

Osaka University, Japan

Reviewed by:

Guang-Zhong Wang,

Shanghai Institute of Nutrition and

Health (CAS), China

Minyoung Jung,

Korea Brain Research Institute, South

Korea

*Correspondence:

Shota Nishitan

nshota@u-fukui.ac.jp

Akemi Tomoda

atomoda@u-fukui.ac.jp

Specialty section: This article was submitted to

Neurogenomics,

a section of the journal

Frontiers in Genetics

Received: 28 October 2021 Accepted: 02 February 2022

Published: 01 March 2022

Citation:

Nishitani S, Kasaba R, Hiraoka D, Shimada K, Fujisawa TX, Okazawa H and Tomoda A (2022) Epigenetic Clock

Deceleration and Maternal

Reproductive Efforts: Associations With Increasing Gray Matter Volume of

the Precuneus.

Front. Genet. 13:803584.

doi: 10.3389/fgene.2022.803584

\section{Epigenetic Clock Deceleration and Maternal Reproductive Efforts: Associations With Increasing Gray Matter Volume of the Precuneus}

\author{
Shota Nishitani ${ }^{1,2,3 *}$, Ryoko Kasaba ${ }^{1,2}$, Daiki Hiraoka ${ }^{1,4}$, Koji Shimada ${ }^{1,2,3,5}$, \\ Takashi X. Fujisawa ${ }^{1,2,3}$, Hidehiko Okazawa ${ }^{3,5}$ and Akemi Tomoda ${ }^{1,2,3,6 *}$
}

${ }^{1}$ Research Center for Child Mental Development, University of Fukui, Fukui, Japan, ${ }^{2}$ Division of Developmental Higher Brain Functions, United Graduate School of Child Development, Hamamatsu University School of Medicine, Osaka University, Kanazawa University, Chiba University, University of Fukui, Osaka, Japan, ${ }^{3}$ Life Science Innovation Center, University of Fukui, Fukui, Japan, ${ }^{4}$ Japan Society for the Promotion of Science, Tokyo, Japan, ${ }^{5}$ Biomedical Imaging Research Center, University of Fukui, Fukui, Japan, ${ }^{6}$ Department of Child and Adolescent Psychological Medicine, University of Fukui Hospital, Fukui, Japan

Reproductive efforts, such as pregnancy, delivery, and interaction with children, make maternal brains optimized for child-rearing. However, extensive studies in non-human species revealed a tradeoff between reproductive effort and life expectancy. In humans, large demographic studies have shown that this is the case for the most part; however, molecular marker studies regarding aging remain controversial. There are no studies simultaneously evaluating the relationship between reproductive effort, aging, and brain structures. We therefore examined the associations between reproductive efforts (parity status, number of deliveries, motherhood period, and cumulative motherhood period), DNA methylation age (mAge) acceleration (based on Horvath's multi-tissue clock and the skin \& blood clock), and the regional gray matter volumes (obtained through brain magnetic resonance imaging (MRI) using voxel-based morphometry) in 51 mothers aged 27-46 years of children in early childhood. We found that increasing reproductive efforts were significantly associated with decelerated aging in mothers with one to four children, even after adjusting for the confounding effects in the multiple linear regression models. We also found that the left precuneus gray matter volume was larger as deceleration of aging occurred; increasing left precuneus gray matter volume, on the other hand, mediates the relationship between parity status and mAge deceleration. Our findings suggest that mothers of children in early childhood, who have had less than four children, may benefit from deceleration of aging mediated via structural changes in the precuneus.

Keywords: DNA methyaltion, imaging epigenetics, longevity, maternal brain, voxel-based morphometry (VBM)

\section{INTRODUCTION}

In non-human species, the established theory (LHT: Life History Theory) is that the greater the reproductive effort, the more finite energy is expended; thus, females tradeoff life expectancy with reproductive effort (Hill and Kaplan, 1999). In humans, several large, population-based demographic studies of more than 140,000 women born between 1820 and 1920 in a preindustrial population in 
Utah, North America, have also shown that women who undertook more reproductive effort lived a shorter lifespan (Penn and Smith, 2007; Bolund et al., 2016). These results are largely in line with the LHT. However, according to Bolund et al. (2016), maternal life expectancy is shortened with four or more births, per the LHT; conversely, deliveries of less than four extended life expectancy (Bolund et al., 2016). Our current monogamous society no longer has an average of nine births (as was common in this previous era); for example, the number of childbirths in first-time married couples who had been married for 15-19 years in Japan was 1.94 in 2015 (The Fifteenth Japanese National Fertility Survey in 2015, 2017). A more recent large, North America-wide demographic study of more than 20,000 women was conducted (Shadyab et al., 2017), which better reflects the current average number of births. Shadyab et al. (2017) reported that the odds ratio (OR) of longevity in Caucasians was higher for mothers who gave birth more than once (two to four children; mean OR: 1.13) compared to that of those who gave birth once (OR: 0.91) or were nulliparous (OR: 1 as reference), even after adjusting for demographic characteristics, socioeconomic status, lifestyle behaviors, reproductive factors, and health-related factors (Shadyab et al., 2017). However, it was consistent with both the LHT and previous population demographics in most aspects, especially regarding having more children; the association between the number of deliveries and longevity was attenuated, and was certainly not higher in those with five or more children. Dior et al. (2013) reported a U-shaped and nonlinear, association between the number of deliveries and all-cause maternal mortality; the lowest risk was at two to three children (Dior et al., 2013). Therefore, while it may be true that having more than four children reduces life expectancy in women, the LHT may not be consistent with births of less than four children.

Hence, recent studies using molecular metrics have attempted to provide biological evidence for the effects of reproductive effort on women's longevity. Telomere length was used as a molecular metric to reflect longevity in the initial study stages. The first study reported that mothers who had more children had shorter telomere lengths (Gray et al., 2014); by contrast, a study of the Mayan tribe in Guatemala reported that mothers who had more children had longer telomeres (Barha et al., 2016). In addition to telomere length, epigenetic age acceleration-which is obtained by calculating the deviation of DNA methylation age (mAge) from chronological age (Horvath, 2013) - has recently attracted attention, serving as a novel biomarker of aging. Using this metric, Ryan et al. showed that in 397 young, 20-22 year-old Filipino women, a higher number of pregnancies was associated with a greater mAge acceleration and shorter telomere length (Ryan et al., 2018). Kresovich et al. also retrospectively used this metric in more than 2,000 women aged 35-74 and living in the US, reporting that increased acceleration of aging was seen with more births (Kresovich et al., 2019). Nevertheless, the results of these studies cannot sufficiently explain why the influence of births of four or fewer children might be exceptionally non-linear. Moreover, the cause of this putative possibility might be difficult to elucidate in retrospective studies on generations that have already completed child-rearing. While the participants in the study by Ryan et al. were supposed to be rearing children, the mean age of childbearing in most developed countries is considerably higher, and was as high as 30.7 years old in Japan in 2016; therefore, while their results are applicable to younger mothers, it remains uncertain whether they can be applied to the general maternal population at present.

In addition to the phenomenon where reproductive effort affects aging and longevity, it has been affirmed that reproductive effort, including the experiences of pregnancy, delivery, and parenting, makes female brain structures more maternal and optimized for parenting (Nishitani et al., 2011; Nishitani et al., 2014; Kim, 2016). Hoekzema et al. (2017) conducted a longitudinal study that followed 25 first-time mothers from time of conception to 2 years after delivery; they found that at 10 months postpartum, the gray matter (GM) volume of the inferior and medial frontal gyrus, superior temporal sulcus, fusiform gyrus, precuneus, and hippocampus-involved in the theory-of-mind network-was reduced compared with during pregnancy (Hoekzema et al., 2017). Zhang et al. (2019) examined a similar longitudinal study and found that mothers showed changes in GM and white matter volumes, as well as the cortical thickness of several regions-including the superior and medial frontal gyrus, insula, limbic lobe, superior and middle temporal gyrus, and precentral gyrus-after 2 years of follow-up (Zhang et al., 2019). Luders et al. (2020) conducted a similar longitudinal study. They reported no decline in areas at 4-6 weeks postpartum compared with the immediate postpartum period (one to two days postpartum); however, there were increased GM volumes in certain regions, including the pre- and postcentral gyrus, thalamus, and precuneus, contrary to Hoekzema et al.'s results (Luders et al., 2020). These studies suggest that reproductive effort affects not only aging, but also elicits changes in brain structure. Contrasting with other species, humans do not reach the end of their life shortly after menopause; in fact, they live nearly twice as long as their age at menopause in this modern era. The grandmother hypothesis, explaining the existence of menopause in human life history by identifying the adaptive value of extended kin networking, has been proposed (Hawkes, 2004), as postmenopausal women have been found to have a great deal of biological and social activity (Kida et al., 2014; Hawkes and Finlay, 2018). This is unique to humans and is considered to be related to humans' high sociability, owing to their highly evolved brains (Hawkes, 2020).

Therefore, we hypothesized that reproductive effort would refine the brain structures involved in the sociability required for parenting, thereby influencing both mAge acceleration and longevity. In the present study, we examined the association between reproductive effort, mAge acceleration measured using salivary DNA, and brain structures evaluated by voxel-based morphometry (VBM), in mothers.

\section{MATERIALS AND METHODS}

\section{Participants}

A total of 57 Japanese biological mothers rearing at least one child of preschool age participated in this study; this dataset is used for 
TABLE 1 | Demographic characteristics of the participants.

\begin{tabular}{|c|c|}
\hline & $(n=51)$ \\
\hline Age (years), Mean (SD) & $35.4(4.4)$ \\
\hline Age (years) at first childbirth & $29.1(4.0)$ \\
\hline Age (years) at last childbirth & $32.3(3.9)$ \\
\hline \multicolumn{2}{|l|}{ Primiparity/Multiparity } \\
\hline Number of deliveries, $n$ (\%) & $11(21.6)$ \\
\hline One & $30(58.8)$ \\
\hline Two & $9(17.6)$ \\
\hline Three & $1(2.0)$ \\
\hline \multicolumn{2}{|l|}{ Four } \\
\hline Motherhood period (age of oldest child) (years) & $6.2(3.2)$ \\
\hline Cumulative motherhood period (years) & $9.9(6.0)$ \\
\hline Exclusive breastfeeding diet, $n(\%)$ & $30(58.8)$ \\
\hline \multicolumn{2}{|l|}{ Household Income (currency = JPY), $n(\%)$} \\
\hline Less than 3 million & $3(5.9)$ \\
\hline 3-5 million & $25(49.0)$ \\
\hline 5-10 million & $21(41.2)$ \\
\hline More than 10 million & $2(3.9)$ \\
\hline \multicolumn{2}{|l|}{ MRI scanner, $n(\%)$} \\
\hline Discovery MR 750 3T/Signa PET/MR 3T & $30(58.8) / 21(41.2)$ \\
\hline Proportion of Epithelial cells (\%) & $15.9(5.8)$ \\
\hline FLANDERS handedness inventory (right/mixed/left) & $48(92.3) / 1(1.9) / 2(3.8)$ \\
\hline PSI (Total/Child/Adult) & $191.5(39.8) / 86.5(18.6) / 105.1(24.6)$ \\
\hline BDI-II & $12.0(9.2)$ \\
\hline
\end{tabular}

PSI, Parenting Stress Index (Abidin, 1995; Namara et al., 1999); BDI-II, The Beck Depression Inventory-Il (Beck, Steer, \& Brown, 1996; Kojima et al., 2002).

our previous studies (Hiraoka et al., 2020; Sakakibara et al., 2021; Kasaba et al., 2021; Shimada et al., 2019). We excluded four mothers who delivered by caesarean section for multiple births, and two mothers of children with disabilities. A total of 51 mothers were included (age range $=27-46$ years; mean age $=$ 34.8 years; standard deviation $(\mathrm{SD})=4.5$ years). Detailed demographics are shown in Table 1. Among the participants, 11 had one child, 30 had two children, nine had three children, and one had four children. We defined "parity status" as primiparity or multiparity, and "number of deliveries" as the number of times a mother gave live birth; these represented the reproductive effort indices. The average age of the oldest children of the participants was 6.2 years $(\mathrm{SD}=3.2)$; this age was defined as the total "motherhood period" until the day of the experiment. We added up the age of all children to calculate an index, referred to as the "cumulative motherhood period"; this reflected the efforts invested in parenting more appropriately for mothers rearing multiple children (Supplementary Figure S1 and Supplementary Table S1). All participants had received at least 12 years of education. Almost all (92.3 \%) were righthanded, according to the FLANDERS handedness inventory (Nicholls et al., 2013; Okubo et al., 2014).

This study's protocol was approved by the Ethics Committee of the University of Fukui (FU20150109 and FU20190107), and was conducted following the Declaration of Helsinki. All participants provided written informed consent for participation in this study.

\section{Questionnaires}

The Japanese version of the Parenting Stress Index (PSI) (Namara et al., 1999) — an adaptation of the PSI (Abidin, 1995) — was used to evaluate the participants' parenting stress (PSI total). The Beck Depression Inventory-II (BDI-II) (Beck et al., 1996; Kojima et al., 2002) was used to measure the participants' depressive symptoms.

\section{DNA Methylation}

Saliva samples were collected using Oragene Discover OGR-500 kits (DNA Genotek Inc., Ottawa, ON, Canada). Saliva DNA was extracted using prepIT ${ }^{\circledR} \bullet 2 \mathrm{P}$ reagent (DNA Genotek Inc.) and was quantified with Qubit ${ }^{\mathrm{TM}}$ dsDNA HS Assay Kit (Thermo Fisher Scientific Inc., Pittsburgh, PA, United States) (Utevsky et al., 2014). Five hundred ng of DNA was bisulfite-treated for cytosine to thymine conversion using the EZ DNA MethylationGold kit (Zymo Research, Irvine, CA, United States). DNA was then whole-genome amplified, fragmented, and hybridized to the HumanMethylationEPIC BeadChip (Illumina Inc., San Diego, CA, United States). The BeadChips were scanned using iSCAN (Illumina Inc.), and the methylation level ( $\beta$ value) was calculated for each queried CpG locus using the GenomeStudio Methylation Module software. As shown in Supplementary Figure S2, a quality check was conducted based on the Psychiatric Genomics Consortium-EWAS quality control pipeline (Ratanatharathorn et al., 2017) using CpGassoc (Barfield et al., 2012), an R package. Samples with probe detection call rates $<90 \%$, and those with an average intensity value of either $<50 \%$ of the experiment-wide sample mean or $<2,000$ arbitrary units were excluded. Probes with detection $p>0.001$, or those based on less than three beads, were set to missing, as were probes that cross-hybridized between autosomes and sex chromosomes. CpG sites with missing data for $>10 \%$ of samples within cohorts were excluded from the analysis. After quality control, 852,775 probes were left for further analysis. 
Horvath's multi-tissue clock mAge (Horvath, 2013) and the skin \& blood clock mAge (Horvath et al., 2018) were calculated based on the online calculator (https://horvath.genetics.ucla.edu/html/ dnamage/). The skin \& blood clock is also known as a novel and highly robust DNAm age estimator for human fibroblasts, keratinocytes, buccal cells, endothelial cells, lymphoblastoid cells, skin, blood, and saliva samples, and has superior accuracy in blood and saliva samples than multi-tissue clock (Horvath et al., 2018). We regressed mAge on chronological age; the unstandardized residuals indicated epigenetic age acceleration. As saliva contains a heterogeneous mixture of cell types that differ in proportion in each sample, using the EpiDISH method (Teschendorff et al., 2017), we estimated the proportion of epithelial cells derived from salivary DNA (Epi) after the preprocessing processes similar to our previous study (Nishitani et al., 2021) and examined whether the proportion of epithelial cells affects mAge acceleration.

\section{MRI Acquisition and Voxel-Based Morphometry}

Image acquisition of 30 participants was performed using a GE Discovery MR 750 3-T scanner (GE Healthcare, Milwaukee, WI). A T1-weighted anatomical dataset was obtained from each subject by a fast-spoiled gradient recalled imaging sequence (voxel size $1 \times 1 \times 1 \mathrm{~mm}, \mathrm{TE}=1.99 \mathrm{~ms}, \mathrm{TR}=6.38 \mathrm{~ms}$, flip angle $=11^{\circ}$ ). Image acquisition of the 21 participants was carried out using a GE Signa PET/MR 3-T scanner (GE Healthcare, Milwaukee, WI). High-resolution structural whole-brain images were acquired using a 3D T1-weighted fast spoiled-gradient recalled imaging sequence (voxel size $1 \times 1 \times 1 \mathrm{~mm}$, TE $=$ $3.24 \mathrm{~ms}, \mathrm{TR}=8.46 \mathrm{~ms}$, flip angle $=11^{\circ}$ ). Given that we used two scanners, a dummy-coded covariate ( 0 vs. 1 ) was added in the analyses. VBM data were analyzed using the Statistical Parametric Mapping software (SPM12; https://www.fil.ion.ucl.ac.uk/spm) implemented in MATLAB 2014a. The T1-weighted images were preprocessed using the VBM approach with modulation, where the images were first segmented into GM, white matter, cerebrospinal fluid, and skull/scalp compartments. Using the iterative high-dimensional normalization approach provided by Diffeomorphic Anatomical Registration through an Exponentiated Lie algebra algorithm (Ashburner, 2007), the segmented GM images were spatially normalized into the stereotaxic space of the Montreal Neurological Institute (MNI). The GM images had an isotropic voxel resolution of 1 . $5 \mathrm{~mm}^{3}$. Any volume change induced by normalization was adjusted via a modulation algorithm. The normalized modulated GM images were spatially smoothed by a Gaussian kernel of 8-mm full-width-at-half-maximum.

\section{Statistical Analysis}

We conducted simple linear regression using heteroscedasticityrobust standard errors, with four types of reproductive effort indices as independent variables to predict mAge acceleration: parity status, number of deliveries, motherhood period, and cumulative motherhood period. We have employed this statistical technique for our analyses since it is no needed the assumption of homoscedasticity. To investigate the extent to which reproductive effort accounts for variance in mAge acceleration in the presence of various potential confounders (chronological age, PSI total, and Epi), multiple regression using robust standard errors was conducted. In the VBM analysis, chronological age, scanner (dummy coded as 0 and 1), and total brain volume were included as covariates of no interest in the design matrix to regress out their effects. The resulting set of voxel values used for each contrast generated a statistical parametric map of the $t$-statistic, SPM $(t)$, which was transformed to a unit normal distribution [SPM $(Z)]$. The statistical threshold was set to $p<0.05$, with family-wise error correction for multiple comparisons at the cluster level (height threshold of $Z>3.09$ ). Significant clusters were localized in the Automated Anatomical Labelling atlases implemented in the MRIcron software package (https://www.nitrc.org/projects/ mricron).

We conducted robust mediation analysis (Alfons et al., 2021) to assess whether the GM volume mediated the link between reproductive efforts and mAge acceleration. We included chronological age as the covariate in the model. The indirect effects of each model were tested by bootstrapping confidence intervals using the robmed package (Alfons et al., 2021). The model parameters were set to give bias-corrected $95 \%$ confidence intervals and to run 5,000 bootstrap resamples. All statistical analyses were performed with R 4.0.5 and SPM 12 .

\section{RESULTS}

\section{Reproductive Effort and Epigenetic Age Acceleration}

Among 353 and $391 \mathrm{CpG}$ sites required to calculate Horvath's multi-tissue clock mAge (Horvath, 2013) and skin \& blood mAge (Horvath et al., 2018), we used 333 and $391 \mathrm{CpG}$ probes, respectively. Seventeen probes were not included in the EPIC array to begin with and three probes (cg19167673, cg27413543, and cg14329157) were removed during data processing. As expected, mAge strongly correlated with chronological age (multi-tissue clock mAge: $R^{2}=0.57, t=8.13, P=1.23 \mathrm{e}-10$; skin \& blood clock mAge: $R^{2}=0.75, t=12.17, P=1.98 \mathrm{e}-16$ ) (Supplementary Figure S3). The median absolute deviation from chronological age was 3.51 years for multi-tissue clock mAge, and 2.47 years for skin \& blood clock mAge. These results were comparable with 2.7 years and 2.5 or 3.0 years, respectively, as reported by Horvath (Horvath, 2013); (Horvath et al., 2018). In simple linear regression analyses, mAge acceleration calculated by the multi-tissue clock was significantly negatively associated with parity status $(\beta=-0.26, t=-2.30, P=0.03)$, number of deliveries $(\beta=-0.29, t=-2.45, P=0.02)$, motherhood period $(\beta=$ $-0.28, t=-2.45, P=0.02)$, and cumulative motherhood period $(\beta$ $=-0.29, t=-2.29, P=0.03$ ) (Table 2 and Figure 1). Conversely, $\mathrm{mAge}$ acceleration calculated by the skin and blood clock was significantly negatively associated with parity status $(\beta=-0.21$, $t=-2.21, P=0.03)$, and number of deliveries $(\beta=-0.28, t=$ $-2.44, P=0.02$ ), while no associations were found with other parameters (Table 2 and Figure 1). In other words, more 
TABLE 2 | Simple linear regression with robust standard errors for mAge accelerations.

\begin{tabular}{|c|c|c|c|c|c|c|}
\hline & \multicolumn{3}{|c|}{ Multi-tissue clock } & \multicolumn{3}{|c|}{ Skin \& blood clock } \\
\hline & $\beta$ & $t$ & $\boldsymbol{P}$ & $\beta$ & $t$ & $\boldsymbol{P}$ \\
\hline \multicolumn{7}{|l|}{ Reproductive effort } \\
\hline Parity status & -0.26 & -2.30 & $0.03^{*}$ & -0.21 & -2.21 & $0.03^{\prime}$ \\
\hline Number of deliveries & -0.29 & -2.45 & $0.02^{*}$ & -0.28 & -2.44 & $0.02^{*}$ \\
\hline Motherhood period & -0.28 & -2.45 & $0.02^{*}$ & -0.13 & -0.92 & 0.36 \\
\hline Cumulative motherhood period & -0.29 & -2.29 & $0.03^{*}$ & -0.25 & -1.84 & 0.07 \\
\hline \multicolumn{7}{|l|}{ Other variables } \\
\hline Age & -0.09 & -0.54 & 0.59 & -0.04 & -0.26 & 0.79 \\
\hline PSI total & -0.42 & -3.15 & $0.003^{\star \star \star}$ & -0.14 & -1.03 & 0.31 \\
\hline BDI-II & -0.29 & -2.14 & $0.04^{*}$ & -0.12 & -0.98 & 0.33 \\
\hline Epi & -0.05 & -0.35 & 0.73 & -0.003 & -0.02 & 0.98 \\
\hline Age at first childbirth & 1.25 & 0.66 & 0.51 & 0.06 & 0.46 & 0.65 \\
\hline Age at last childbirth & -0.64 & -0.36 & 0.72 & 0.02 & 0.18 & 0.86 \\
\hline Exclusive breastfeeding diet & 0.09 & 0.59 & 0.56 & 0.24 & 1.93 & 0.06 \\
\hline Household income & 0.04 & 0.27 & 0.79 & 0.18 & 1.44 & 0.16 \\
\hline
\end{tabular}

${ }^{* * * *} P<0.05,{ }^{* *} P<0.01, P<0.005$.

BDI-II, The Beck Depression Inventory-II; mAge, DNA, methylation age; PSI, parenting stress index.

Multi-tissue clock

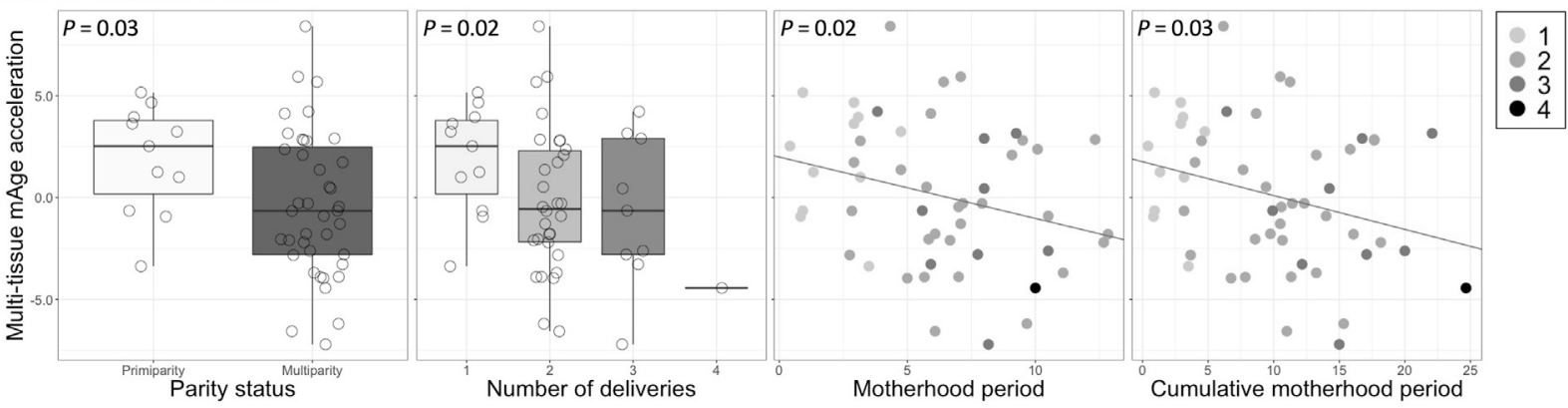

Skin \& blood clock
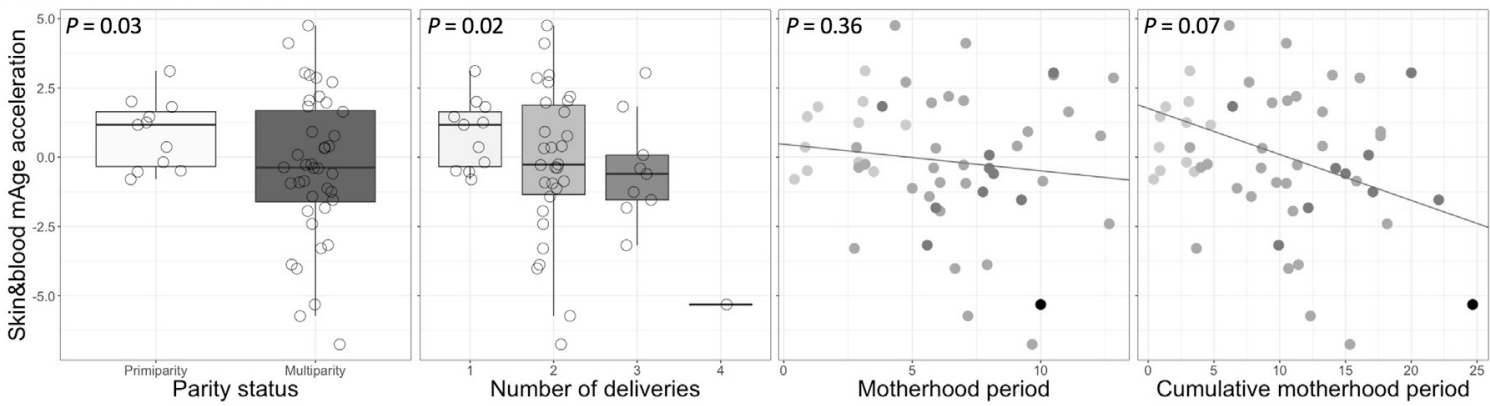

FIGURE 1 | Associations between each reproductive effort and mAge acceleration. Top: multi-tissue clock; bottom: skin \& blood clock. The gray shading in the legend on the upper right reflects the number of deliveries.

reproductive efforts showed diminished mAge acceleration (Figure 1). PSI total $(\beta=-0.42, t=-3.15, P=0.003)$ and BDI-II $(\beta=-0.29, t=-2.14, P=0.04)$ were significantly associated with mAge acceleration calculated by the multitissue clock. No other demographic characteristics were associated with mAge acceleration for both calculations (Table 2). The correlation coefficients matrix between all the covariate combinations used in the analysis are shown in Supplementary Figure S4.
In addition to the simple linear regression, we also conducted multiple linear regression analyses to assess whether the other variables (chronological age, PSI total, and Epi) confounded the results (Table 3). Although chronological age and Epi were not significantly associated with mAge accelerations in the simple linear regression analysis, they were added in the multiple linear regression models as covariates. We chose PSI total instead of BDI-II as a covariate to avoid multi-collinearity issue in the regression model, as the two demonstrated a high correlation 
TABLE 3 | Multiple linear regression with robust standard errors for mAge accelerations.

\begin{tabular}{|c|c|c|c|c|c|c|c|c|c|}
\hline & & \multicolumn{4}{|c|}{ Multi-tissue clock } & \multicolumn{4}{|c|}{ Skin \& blood clock } \\
\hline & & $\begin{array}{l}\text { Parity } \\
\text { status }\end{array}$ & $\begin{array}{c}\text { Number } \\
\text { of } \\
\text { deliveries }\end{array}$ & $\begin{array}{l}\text { Motherhood } \\
\text { period }\end{array}$ & $\begin{array}{c}\text { Cumulative } \\
\text { motherhood } \\
\text { period }\end{array}$ & $\begin{array}{l}\text { Parity } \\
\text { status }\end{array}$ & $\begin{array}{c}\text { Number } \\
\text { of } \\
\text { deliveries }\end{array}$ & $\begin{array}{l}\text { Motherhood } \\
\text { period }\end{array}$ & $\begin{array}{c}\text { Cumulative } \\
\text { motherhood } \\
\text { period }\end{array}$ \\
\hline \multirow{3}{*}{$\begin{array}{l}\text { Reproductive } \\
\text { effort }\end{array}$} & $\beta$ & -0.25 & -0.26 & -0.29 & -0.28 & -0.21 & -0.27 & -0.14 & -0.27 \\
\hline & $t$ & -2.26 & -2.52 & -2.15 & -2.14 & -2.23 & -2.36 & -0.91 & -2.09 \\
\hline & $P$ & $0.03^{\star}$ & $0.02^{\star}$ & $0.04^{*}$ & $0.04^{*}$ & $0.03^{*}$ & $0.02^{*}$ & 0.37 & $0.04^{\star}$ \\
\hline \multirow[t]{3}{*}{ Age } & $\beta$ & 0.07 & 0.02 & 0.14 & 0.10 & 0.05 & 0.01 & 0.06 & 0.09 \\
\hline & $t$ & 0.43 & 0.15 & 0.74 & 0.61 & 0.34 & 0.08 & 0.38 & 0.635 \\
\hline & $P$ & 0.67 & 0.88 & 0.46 & 0.54 & 0.73 & 0.94 & 0.70 & 0.53 \\
\hline \multirow[t]{3}{*}{ PSI total } & $\beta$ & -0.42 & -0.41 & -0.41 & -0.42 & -0.14 & -0.13 & -0.14 & -0.14 \\
\hline & $t$ & -3.06 & -3.23 & -3.05 & -0.32 & -0.96 & -0.90 & -0.97 & -0.96 \\
\hline & $P$ & $0.004^{\star \star \star}$ & $0.002^{\star \star \star}$ & $0.004^{\star \star \star}$ & $0.003^{\star \star \star}$ & 0.34 & 0.37 & 0.34 & 0.34 \\
\hline \multirow[t]{3}{*}{ Epi } & $\beta$ & 0.03 & 0.03 & 0.06 & 0.06 & 0.04 & 0.03 & 0.04 & 0.06 \\
\hline & $t$ & 0.24 & 0.20 & 0.42 & 0.41 & 0.24 & 0.22 & 0.27 & 0.39 \\
\hline & $P$ & 0.81 & 0.85 & 0.68 & 0.69 & 0.81 & .83 & 0.79 & 0.70 \\
\hline \multirow[t]{3}{*}{ Model } & $R^{2}$ & 0.16 & 0.18 & 0.17 & 0.17 & -0.02 & 0.015 & -0.05 & 0.004 \\
\hline & $F$ & 4.99 & 4.84 & 3.66 & 3.98 & 1.98 & 1.86 & 0.46 & 1.48 \\
\hline & $P$ & $0.002^{\star \star \star}$ & $0.002^{\star \star \star}$ & $0.01^{*}$ & $0.007^{\star \star}$ & 0.11 & 0.13 & 0.77 & 0.22 \\
\hline
\end{tabular}

${ }^{* * * *} P<0.05,{ }^{* *} P<0.01, P<0.005$

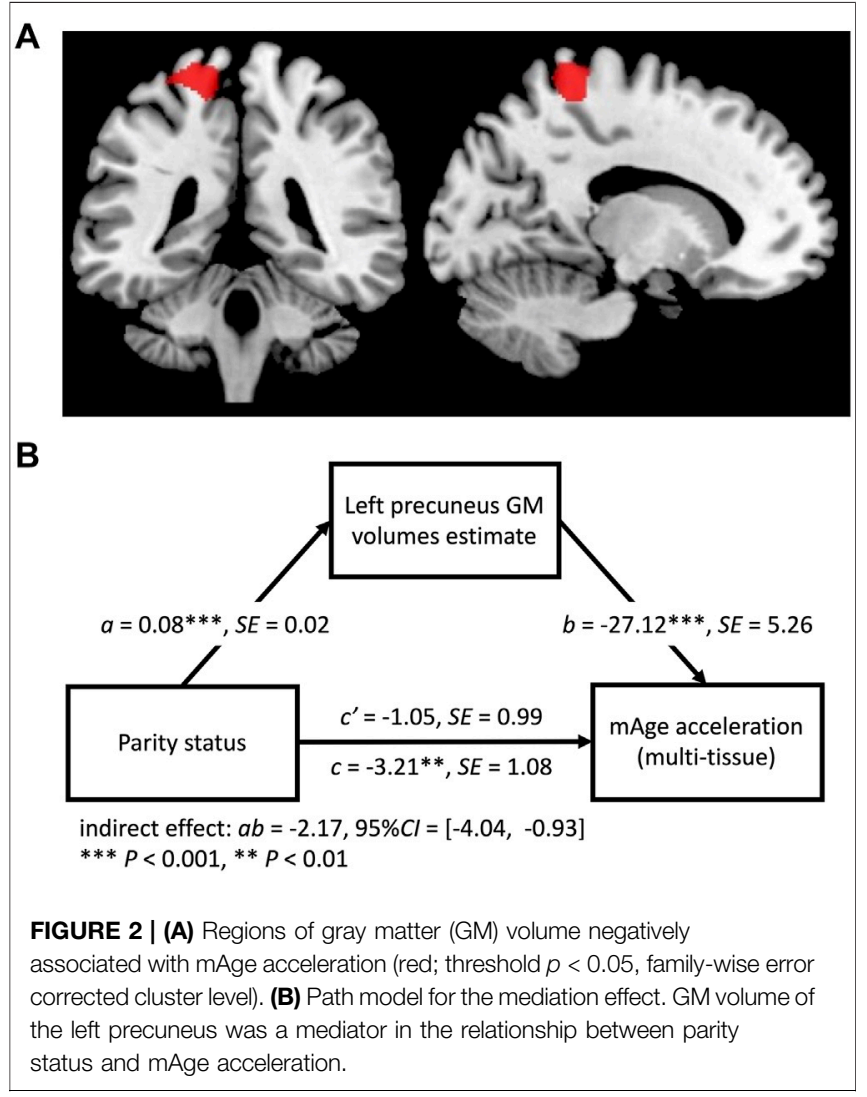

( $r=0.72, P=2.85 \mathrm{e}-09)$; additionally, the association between PSI total and mAge acceleration was greater than that of BDI-II (Table 2). Results showed that all four reproductive effort indices were significantly negatively associated with mAge acceleration calculated by the multi-tissue clock, even after adjusting for the confounding effects $(P s<0.05$, Table 3). Although the results of
mAge acceleration calculated by the skin \& blood clock showed significant associations with parity status $(P=0.03)$, number of deliveries $(P=0.02)$, and cumulative motherhood period $(P=0.04)$ in the multiple linear regression model, these models were not significant (Table 3). Hence, we used mAge acceleration based on the multi-tissue clock for subsequent analyses.

\section{VBM and Path Analysis}

The VBM results showed that mAge acceleration was negatively correlated with GM volume within a cluster in the left precuneus (Montreal Neurological Institute [MNI] coordinates: $x=-17, y=$ $-39, z=68$; cluster size $=727$ voxels; $P=0.04$, family-wise error [FWE] corrected cluster level; Figure 2A). There was a significant indirect effect of the precuneus GM volume on parity status and mAge acceleration (indirect effect $=-2.17$, 95\% Confidence Interval $=[-4.04,-0.93]$; Figure $2 \mathbf{B})$. No other reproductive efforts had a significant indirect effect.

\section{DISCUSSION}

This study examined the relationship between reproductive effort, mAge acceleration, and brain structure in mothers of children in early childhood. Our results showed that their reproductive effort-regarding parity status, number of deliveries, motherhood period, and cumulative motherhood period-was associated with mAge deceleration, even after adjusting for potential confounders. Although previous studies have examined the relationship between mAge acceleration or telomere length and the number of deliveries as a representative variable, this is the first time that mAge acceleration has been simultaneously examined in relation to the other reproductive effort indices, also considering daily parenting stress. We also found that the precuneus GM volume increased as mAge deceleration occurred. We also observed a mediation effect of 
greater left precuneus GM volume on the relationship between parity status and mAge deceleration. This suggests that the precuneus - a central node in the human brain that supports complex cognition and behavior-may be associated with age deceleration in child-rearing mothers. This also suggests that multiparity between two to four births lead to maternal brain changes in the left precuneus, which contributes more to mAge deceleration than just one birth.

Our results were limited as we only included mothers who gave birth to one to four children; thus, we do not know how this would have affected women with more than four births. Still, among mothers who gave birth to one to four children, our results seemed to contradict the LHT (Hill and Kaplan, 1999). Our results suggest that mothers who gave birth to less than four children and have currently been rearing them have aged more slowly, depending on their reproductive efforts. Our findings are consistent with previous large demographic studies reporting that deliveries of less than four children extended, rather than diminished, life expectancy (Dior et al., 2013; Bolund et al., 2016; Shadyab et al., 2017). We speculate that this avoids a downward trend in the birth rate, since having only one offspring born to each couple would lead to a decline in population in a monogamous society. While childbearing certainly comes at the cost of energy based on the LHT, giving birth to two or more children while having fewer than four may benefit the mother's lifespan, even accounting for the tradeoffs of childbirth.

One possible biological cause underlying this phenomenon could be due to maternal brain alteration. Human mothers' brains undergo dynamic structural and functional changes during pregnancy and the early postpartum period to facilitate their psychological and behavioral adaptation to parenting (Kim, 2016; Hoekzema et al., 2017; Zhang et al., 2019; Luders et al., 2020). This transition to the maternal brain seems to particularly enhance the functioning of the reward, social information, and emotion regulation circuits (Ho et al., 2014). We identified that mAge deceleration associated with parity status was linked to an increase in precuneus GM volume in mothers, which is similar to the findings of the study by Luders et al. where an increase in precuneus GM volume was observed at 4-6 weeks postpartum, compared with 1-2 days postpartum (Luders et al., 2020). The precuneus is included in the social information circuit and is a hub for the default mode network (DMN), which involves empathy, as well as self-monitoring and reflection (Utevsky et al., 2014). In other words, becoming a mother of two or more children may have led to more complex social interactions, which may have contributed to a greater precuneus GM volume than in the past. It has also been found that patients with Alzheimer's disease $(\mathrm{AD})$ and in the prodromal stage of $\mathrm{AD}$ have decreased $\mathrm{DMN}$ functions and precuneus GM volume compared to controls (Goto et al., 2015). Using postmortem brains, a telomere length analysis of each brain region comprising the DMN showed that the precuneus telomere length was shorter in $\mathrm{AD}$ and the prodromal stage of $\mathrm{AD}$ than in controls, whereas there were no differences in the frontal, inferior temporal, posterior cingulate gyrus or visual cortex (Mahady et al., 2020). Furthermore, this telomere length reduction in the precuneus also correlated with cognitive task performance. One of the main symptoms of PTSD is an elevated rumination characterized by repetitive, negative self-focused cognition; it has been reported that reduced functional connectivity between the isthmus cingulate and the left precuneus within the DMN is linked to this high level of rumination (Philippi et al., 2020). Lifetime trauma burden and suffering from both current and lifetime PTSD have been reported to cause GrimAge acceleration (Katrinli et al., 2020). These studies support the notion that aging and mental stress that accelerates aging may affect the functional connectivity between the precuneus and the other brain network region. The sophistication of the social information and DMN functions involved in becoming a mother may have contributed to the mAge deceleration via enhancing precuneus function and volume; however, this finding is not related to the number of deliveries-which accounts for the major reproductive effort-but rather to parity status (whether a baby is born to a mother once or twice or more), and is not a model that is valid as the number of deliveries increases.

Our results were partially inconsistent with previous studies. Ryan et al. reported mAge acceleration in mothers who had given birth to one to five children, as well as in nulliparous women (Ryan et al., 2018). However, their study participants were 20-22 years-old, which is relatively young compared with the average age of a primipara in modern, developed countries. Some reports suggest that a higher age at first birth is associated with longer telomere length (Fagan et al., 2017) and longer life expectancy (Shadyab et al., 2017). Thus, the association may be linear, rather than U-shaped, when the age at first birth is relatively young, as in this previous study. In addition, various environmental factors have been reported to influence mAge acceleration, most notably stress (Hoare et al., 2020; Katrinli et al., 2020). While Ryan et al. adjusted for socioeconomic status, they did not consider the effects of psychological aspects, such as parental stress; therefore, an analysis adjusting for these effects may also be necessary. In our results, higher parental stress, not only reproductive effort, was associated with the deceleration of aging. Conversely, Kresovich et al. reported accelerated aging in mothers who gave birth to one to four or more children, as well as in nulliparous women (Kresovich et al., 2019). However, their study was retrospective and included those who were currently out of the child-rearing phase (an average age of 55 years when the blood samples were collected), which is different from the present study, involving mothers of children in early childhood. Their results may thus be confounded by menopause (Gray et al., 2014) and the higher genetic risks for developing breast cancer, which is a unique characteristic of this cohort population (Sandler et al., 2017). By contrast, Barha et al. found longer telomeres in mothers who gave birth to one to six babies (Barha et al., 2016). Although this study did not measure mAge acceleration, telomere length results indicate that age deceleration may have occurred, perhaps akin to our findings. Additionally, our studies were similar in that the DNA was saliva-derived. Our method of estimating mAge by using Horvath's multi-tissue clock should not be noticeably affected, even if the tissue from which the DNA was derived was different (Horvath, 2013). Still, the similarities regarding the type of sample may not be particularly relevant. Additionally, the participants in their study were, on average, 
39.4 years old, premenopausal, and probably still rearing their children. This consistency in age and rearing young children may be related to the directional consistency of the two results. Although their results were linearly regressed, on closer inspection, an inverted U-shaped approximation with a peak at two to three births appears to be more appropriate.

There are at least six potential limitations concerning our study's results. First, the small number of participants; we only had one participant with more than four children, limiting the ability to observe the U-shaped association that we hypothesized. Small group sizes may preclude drawing significant conclusions and limit generalizability of the results; thus, we will confirm these pilot results by increasing the sample size in future studies. Second, there were no nulliparous participants. Shadyab et al. (2017) reported that mothers had a longer life expectancy than nulliparous women (Shadyab et al., 2017). It is also known that changes in brain morphology and cognitive function occur when women become mothers (Hoekzema et al., 2017; Zhang et al., 2019; Luders et al., 2020); therefore, it is necessary to include nulliparous women to show the association between mAge acceleration and precuneus GM volume with brain materialization. Third, we used saliva DNA samples for mAge and mAge acceleration calculation. This limits the use of other newly developed blood DNA composite biomarkers, including: PhenoAge, which more effectively captures the epigenetic biomarkers of physiological age and discriminates between morbidity and mortality more definitively in individuals of the same chronological age (Levine et al., 2018); and GrimAge-named after the Grim Reaper-which predicts lifespan and healthspan in units of years (Lu et al., 2019). However, Horvath's multi-tissue clock can be used for any type of tissues, enabling a direct comparison with most previous studies based on blood DNA (Ryan et al., 2018; Kresovich et al., 2019). Forth, there was a significant association that higher the PSI, the more age deceleration based on the multi-tissue clock $(p=0.003)$, but not for skin \& blood clock ( $p=0.31)$. Zannas et al. (2015) have reported that lifetime stress accelerates epigenetic aging since the multi-tissue clock has 85 probes located within glucocorticoid response elements (Zannas et al., 2015). Thus, these specific probes included in the multi-tissue clock might have affected the association with PSI. However, the effect directionality of our results was different. This may suggest that the age deceleration was not effortlessly obtained as it was also associated with the length of the motherhood period. Fifth, we do not have information whether these participants cohabit with their parent or parent-in-law. According to the public demographics, nuclear families in Japan were $82.7 \%$ (2016) (Graphical Review of Japanese Household, 2016) and this might have influenced the results. Finally, we have focused only on mAge acceleration, but it is necessary to explore the relationship with the genome-wide profile, which is a future research goal.

\section{CONCLUSION}

Despite these limitations, our results suggest that reproductive effort in mothers may refine the brain structures involved in the sociability required for parenting, conversely influencing mAge acceleration and longevity. Our results also suggest that the mAge deceleration associated with changes in the precuneus may be one of the phenomena linked to the maternalization of the brain.

\section{DATA AVAILABILITY STATEMENT}

The original contributions presented in the study are included in the article/Supplementary Material, further inquiries can be directed to the corresponding authors.

\section{ETHICS STATEMENT}

The studies involving human participants were reviewed and approved by The Ethics Committee of the University of Fukui. The patients/participants provided their written informed consent to participate in this study.

\section{AUTHOR CONTRIBUTIONS}

SN and AT conceived and designed the project. SN, RK, DH, KS, TXF, and HO performed the experiments, collected, and analyzed the data. SN drafted the manuscript.

\section{FUNDING}

All phases of this study were supported by AMED under Grant Number JP20gk0110052 (AT and SN), JSPS KAKENHI Scientific Research (A) (JP19H00617 to AT), Scientific Research (C) (JP20K02700 to SN), Grant-in-Aid for "Creating a Safe and Secure Living Environment in the Changing Public and Private Spheres" from the Japan Science and Technology Agency (JST)/Research Institute of Science and Technology for Society (RISTEX), Research grant from Japan-United States Brain Research Cooperative Program (AT), Research Grants from the University of Fukui (FY 2019 and 2020 to SN), Grant-in-Aid for Translational Research from the Life Science Innovation Center, University of Fukui (LSI20305 to SN), and Grant for Life Cycle Medicine from Faculty of Medical Sciences, University of Fukui (SN).

\section{ACKNOWLEDGMENTS}

We would like to thank all the participants and the staff at the Research Center for Child Mental Development.

\section{SUPPLEMENTARY MATERIAL}

The Supplementary Material for this article can be found online at: https://www.frontiersin.org/articles/10.3389/fgene.2022.803584/ full\#supplementary-material 


\section{REFERENCES}

Abidin, R. R. (1995). Parenting Stress Index (PSI), Manual. 3rd ed. Charlottesville, VA: Pediatric Psychology Press.

Alfons, A., Ates, N. Y., and Groenen, P. J. F. (2021). A Robust Bootstrap Test for Mediation Analysis, 2021 for Mediation Analysis. Organizational Res. Methods press. doi:10.1177/1094428121999096

Ashburner, J. (2007). A Fast Diffeomorphic Image Registration Algorithm. Neuroimage 38, 95-113. doi:10.1016/j.neuroimage.2007.07.007

Barfield, R. T., Kilaru, V., Smith, A. K., and Conneely, K. N. (2012). CpGassoc: an R Function for Analysis of DNA Methylation Microarray Data. Bioinformatics 28, 1280-1281. doi:10.1093/bioinformatics/bts124

Barha, C. K., Hanna, C. W., Salvante, K. G., Wilson, S. L., Robinson, W. P., Altman, R. M., et al. (2016). Number of Children and Telomere Length in Women: A Prospective, Longitudinal Evaluation. PLoS One 11, e0146424. doi:10.1371/ journal.pone.0146424

Beck, A. T., Steer, R. A., and Brown, G. K. (1996). Manual for the Beck Depression Inventory-II. San Antonio, TX: Psychological Corporation.

Bolund, E., Lummaa, V., Smith, K. R., Hanson, H. A., and Maklakov, A. A. (2016). Reduced Costs of Reproduction in Females Mediate a Shift from a Male-Biased to a Female-Biased Lifespan in Humans. Sci. Rep. 6, 24672. doi:10.1038/ srep24672

Dior, U. P., Hochner, H., Friedlander, Y., Calderon-Margalit, R., Jaffe, D., Burger, A., et al. (2013). Association between Number of Children and Mortality of Mothers: Results of a 37-year Follow-Up Study. Ann. Epidemiol. 23, 13-18. doi:10.1016/j.annepidem.2012.10.005

Fagan, E., Sun, F., Bae, H., Elo, I., Andersen, S. L., Lee, J., et al. (2017). Telomere Length Is Longer in Women with Late Maternal Age. Menopause 24, 497-501. doi:10.1097/gme.0000000000000795

Goto, M., Abe, O., Aoki, S., Hayashi, N., Ohtsu, H., Takao, H., et al. (2015). Longitudinal gray-matter Volume Change in the Default-Mode Network: Utility of Volume Standardized with Global gray-matter Volume for Alzheimer's Disease: a Preliminary Study. Radiol. Phys. Technol. 8, 64-72. doi:10.1007/s12194-014-0295-9

Graphical Review of Japanese Household, from Comprehensive Survey of Living Conditions, Ministry of Health Labour and Welfare, Tokyo, 2016.

Gray, K. E., Schiff, M. A., Fitzpatrick, A. L., Kimura, M., Aviv, A., and Starr, J. R. (2014). Leukocyte Telomere Length and Age at Menopause. Epidemiology 25, 139-146. doi:10.1097/ede.0000000000000017

Hawkes, K. (2020). Cognitive Consequences of Our Grandmothering Life History: Cultural Learning Begins in Infancy. Phil. Trans. R. Soc. B 375, 20190501. doi:10.1098/rstb.2019.0501

Hawkes, K., and Finlay, B. L. (2018). Mammalian Brain Development and Our Grandmothering Life History. Physiol. Behav. 193, 55-68. doi:10.1016/j. physbeh.2018.01.013

Hawkes, K. (2004). The Grandmother Effect. Nature 428, 128-129. doi:10.1038/ $428128 \mathrm{a}$

Hill, K., and Kaplan, H. (1999). Life History Traits in Humans: Theory and Empirical Studies. Annu. Rev. Anthropol. 28, 397-430. doi:10.1146/annurev. anthro.28.1.397

Hiraoka, D., Nishitani, S., Shimada, K., Kasaba, R., Fujisawa, T. X., and Tomoda, A. (2020). Epigenetic Modification of the Oxytocin Gene Is Associated with gray Matter Volume and Trait Empathy in Mothers. Psychoneuroendocrinology 123, 105026. doi:10.1016/j.psyneuen.2020.105026

Ho, S. S., Konrath, S., Brown, S., and Swain, J. E. (2014). Empathy and Stress Related Neural Responses in Maternal Decision Making. Front. Neurosci. 8, 152. doi: $10.3389 /$ fnins.2014.00152

Hoare, J., Stein, D. J., Heany, S. J., Fouche, J.-P., Phillips, N., Er, S., et al. (2020). Accelerated Epigenetic Aging in Adolescents from Low-Income Households Is Associated with Altered Development of Brain Structures. Metab. Brain Dis. 35, 1287-1298. doi:10.1007/s11011-020-00589-0

Hoekzema, E., Barba-Müller, E., Pozzobon, C., Picado, M., Lucco, F., GarcíaGarcía, D., et al. (2017). Pregnancy Leads to Long-Lasting Changes in Human Brain Structure. Nat. Neurosci. 20, 287-296. doi:10.1038/nn.4458

Horvath, S. (2013). DNA Methylation Age of Human Tissues and Cell Types. Genome Biol. 14, R115. doi:10.1186/gb-2013-14-10-r115
Horvath, S., Oshima, J., Martin, G. M., Lu, A. T., Quach, A., Cohen, H., et al. (2018) Epigenetic Clock for Skin and Blood Cells Applied to Hutchinson Gilford Progeria Syndrome and Ex Vivo Studies. Aging 10, 1758-1775. doi:10.18632/ aging.101508

Kasaba, R., Shimada, K., and Tomoda, A. (2021). Neural Mechanisms of Parental Communicative Adjustments in Spoken Language. Neuroscience 457, 206-217. doi:10.1016/j.neuroscience.2020.12.002

Katrinli, S., Stevens, J., Wani, A. H., Lori, A., Kilaru, V., van Rooij, S. J. H., et al. (2020). Evaluating the Impact of Trauma and PTSD on Epigenetic Prediction of Lifespan and Neural Integrity. Neuropsychopharmacol. 45, 1609-1616. doi:10. 1038/s41386-020-0700-5

Kida, T., Nishitani, S., Tanaka, M., Takamura, T., Sugawara, M., and Shinohara, K. (2014). I Love My Grandkid! an NIRS Study of Grandmaternal Love in Japan. Brain Res. 1542, 131-137. doi:10.1016/j.brainres.2013.10.028

Kim, P. (2016). Human Maternal Brain Plasticity: Adaptation to Parenting. New Dir. Child Adolesc. Develop. 2016, 47-58. doi:10.1002/cad.20168

Kojima, M., Furukawa, T. A., Takahashi, H., Kawai, M., Nagaya, T., and Tokudome, S. (2002). Cross-cultural Validation of the Beck Depression Inventory-II in Japan. Psychiatry Res. 110, 291-299. doi:10.1016/s01651781(02)00106-3

Kresovich, J. K., Harmon, Q. E., Xu, Z., Nichols, H. B., Sandler, D. P., and Taylor, J. A. (2019). Reproduction, DNA Methylation and Biological Age. Hum. Reprod. 34, 1965-1973. doi:10.1093/humrep/dez149

Levine, M. E., Lu, A. T., Quach, A., Chen, B. H., Assimes, T. L., Bandinelli, S., et al. (2018). An Epigenetic Biomarker of Aging for Lifespan and Healthspan. Aging 10, 573-591. doi:10.18632/aging.101414

Lu, A. T., Quach, A., Wilson, J. G., Reiner, A. P., Aviv, A., Raj, K., et al. (2019). DNA Methylation GrimAge Strongly Predicts Lifespan and Healthspan. Aging 11, 303-327. doi:10.18632/aging.101684

Luders, E., Kurth, F., Gingnell, M., Engman, J., Yong, E.-L., Poromaa, I. S., et al. (2020). From Baby Brain to Mommy Brain: Widespread gray Matter Gain after Giving Birth. Cortex 126, 334-342. doi:10.1016/j.cortex.2019.12.029

Mahady, L., He, B., Malek-Ahmadi, M., and Mufson, E. J. (2020). Telomeric Alterations in the Default Mode Network during the Progression of Alzheimer's Disease: Selective Vulnerability of the Precuneus. Neuropathol. Appl. Neurobiol. 47 (3), 428-440. doi:10.1111/nan.12672

The Fifteenth Japanese National Fertility Survey in 2015 (2017). Marriage Process and Fertility of Married Couples Attitudes toward Marriage and Family Among Japanese Singles. Natl. Inst. Popul. Soc. Security Res. (Japan), 1-26. https://www. ipss.go.jp/ps-doukou/e/doukou15/Nfs15_points_eng.pdf.

Namara, M., Kanematsu, Y., Araki, A., Maru, M., Nakamura, N., Takeda, J., et al. (1999). Validity and Reliability of the Japanese Version of the Parenting Stress Index. J. Child Health 58 (5), 610-616.

Nicholls, M. E. R., Thomas, N. A., Loetscher, T., and Grimshaw, G. M. (2013). The Flinders Handedness Survey (FLANDERS): a Brief Measure of Skilled Hand Preference. Cortex 49, 2914-2926. doi:10.1016/j.cortex.2013.02.002

Nishitani, S., Doi, H., Koyama, A., and Shinohara, K. (2011). Differential Prefrontal Response to Infant Facial Emotions in Mothers Compared with Non-mothers. Neurosci. Res. 70, 183-188. doi:10.1016/j.neures.2011.02.007

Nishitani, S., Kuwamoto, S., Takahira, A., Miyamura, T., and Shinohara, K. (2014). Maternal Prefrontal Cortex Activation by Newborn Infant Odors. Chem. Senses 39, 195-202. doi:10.1093/chemse/bjt068

Nishitani, S., Suzuki, S., Ochiai, K., Yao, A., Fujioka, T., Fujisawa, T. X., et al. (2021). Altered Epigenetic Clock in Children Exposed to Maltreatment. Psychiatry Clin. Neurosci. 75, 110-112. doi:10.1111/pcn.13183

Okubo, M., Suzuki, H., and Nicholls, M. E. R. (2014). A Japanese Version of the FLANDERS Handedness Questionnaire. Jpn. J. Psychol. 85, 474-481. doi:10. 4992/jjpsy.85.13235

Penn, D. J., and Smith, K. R. (2007). Differential Fitness Costs of Reproduction between the Sexes. Proc. Natl. Acad. Sci. 104, 553-558. doi:10.1073/pnas.0609301103

Philippi, C. L., Pessin, S., Reyna, L., Floyd, T., and Bruce, S. E. (2020). Cortical Midline Structures Associated with Rumination in Women with PTSD. J. Psychiatr. Res. 131, 69-76. doi:10.1016/j.jpsychires.2020.09.001

Ratanatharathorn, A., Boks, M. P., Maihofer, A. X., Aiello, A. E., Amstadter, A. B., Ashley-Koch, A. E., et al. (2017). Epigenome-wide Association of PTSD from Heterogeneous Cohorts with a Common Multi-Site Analysis Pipeline. Am. J. Med. Genet. 174, 619-630. doi:10.1002/ajmg.b.32568 
Ryan, C. P., Hayes, M. G., Lee, N. R., McDade, T. W., Jones, M. J., Kobor, M. S., et al. (2018). Reproduction Predicts Shorter Telomeres and Epigenetic Age Acceleration Among Young Adult Women. Sci. Rep. 8, 11100. doi:10.1038/ s41598-018-29486-4

Sakakibara, N., Makita, K., Hiraoka, D., Kasaba, R., Kuboshita, R., Shimada, K., et al. (2021). Increased Resting-state Activity in the Cerebellum with Mothers Having Less Adaptive Sensory Processing and Trait Anxiety. Hum. Brain Mapp. 42, 4985-4995. doi:10.1002/hbm.25594

Sandler, D. P., Hodgson, M. E., Deming-Halverson, S. L., Juras, P. S., D'Aloisio, A. A., Suarez, L. M., et al. (2017). The Sister Study Cohort: Baseline Methods and Participant Characteristics. Environ. Health Perspect. 125, 127003. doi:10.1289/ ehp1923

Shadyab, A. H., Gass, M. L. S., Stefanick, M. L., Waring, M. E., Macera, C. A., Gallo, L. C., et al. (2017). Maternal Age at Childbirth and Parity as Predictors of Longevity Among Women in the United States: The Women's Health Initiative. Am. J. Public Health 107, 113-119. doi:10.2105/ajph.2016.303503

Shimada, K., Kasaba, R., Yao, A., and Tomoda, A. (2019). Less Efficient Detection of Positive Facial Expressions in Parents at Risk of Engaging in Child Physical Abuse. BMC Psychol. 7, 56. doi:10.1186/s40359-019-0333-9

Teschendorff, A. E., Breeze, C. E., Zheng, S. C., and Beck, S. (2017). A Comparison of Reference-Based Algorithms for Correcting Cell-type Heterogeneity in Epigenome-wide Association Studies. BMC Bioinformatics 18, 105. doi:10. 1186/s12859-017-1511-5

Utevsky, A. V., Smith, D. V., and Huettel, S. A. (2014). Precuneus Is a Functional Core of the Default-Mode Network. J. Neurosci. 34, 932-940. doi:10.1523/ jneurosci.4227-13.2014
Zannas, A. S., Arloth, J., Carrillo-Roa, T., Iurato, S., Röh, S., Ressler, K. J., et al. (2015). Lifetime Stress Accelerates Epigenetic Aging in an Urban, African American Cohort: Relevance of Glucocorticoid Signaling. Genome Biol. 16, 266. doi:10.1186/s13059-015-0828-5

Zhang, K., Wang, M., Zhang, J., Du, X., and Chen, Z. (2019). Brain Structural Plasticity Associated with Maternal Caregiving in Mothers: A Voxel- and Surface-Based Morphometry Study. Neurodegener Dis. 19, 192-203. doi:10. $1159 / 000506258$

Conflict of Interest: The authors declare that the research was conducted in the absence of any commercial or financial relationships that could be construed as a potential conflict of interest.

Publisher's Note: All claims expressed in this article are solely those of the authors and do not necessarily represent those of their affiliated organizations, or those of the publisher, the editors and the reviewers. Any product that may be evaluated in this article, or claim that may be made by its manufacturer, is not guaranteed or endorsed by the publisher.

Copyright (c) 2022 Nishitani, Kasaba, Hiraoka, Shimada, Fujisawa, Okazawa and Tomoda. This is an open-access article distributed under the terms of the Creative Commons Attribution License (CC BY). The use, distribution or reproduction in other forums is permitted, provided the original author(s) and the copyright owner(s) are credited and that the original publication in this journal is cited, in accordance with accepted academic practice. No use, distribution or reproduction is permitted which does not comply with these terms. 